\title{
Managing pediatric osteoporosis
}

\section{"[In order] to improve pediatric bone health, which has lifelong implications, a correct evaluation of the etiology and pathogenesis of diseases, and the role of diseases and/or treatments on bone health, are mandatory."}

\section{KEYWORDS: bone mineral density $\approx$ children $\approx$ fragility fractures $\approx$ osteopenia - osteoporosis - primary osteoporosis $=$ secondary osteoporosis}

Pediatric osteoporosis (PO) is a condition that is increasingly recognized, rarely due to primary genetic diseases of the bone, but more frequently a consequence of chronic illness and/or treatments [1,2].

Considering dual-energy $\mathrm{x}$-ray absorptiometry as the gold standard to evaluate bone status, the relationship between bone mineral density (BMD) and risk of fracture in children is unknown; therefore, it is not possible to define thresholds below which there is an established increased risk. Furthermore, BMD measurements in children are affected by body size as the bone density value produced is an areal bone density (aBMD), failing to provide a volumetric BMD (vBMD). So, a child who is short for their age may receive an inappropriate diagnosis of osteoporosis [3]. Normal BMD values now exist for children aged 7 years and older [4], and there is also a position statement on bone densitometry in children, which states that $\mathrm{PO}$ diagnosis should be made on the basis of a low BMD or bone mineral content ( $\mathrm{z}$-score $\leq-2.0$, adjusted for age, gender and body size), and the presence of a clinically significant fracture history [5].

\section{Causes of pediatric osteoporosis}

Genetic factors play an essential role in the pathogenesis of PO. Many genes have been discovered as possible candidates for the regulation of bone mass [6]; however, the mechanisms that underlie the association between given genes and osteoporosis are poorly understood [1,2]. An essential point for improving bone health is to reach a high peak bone mass (PBM). Even if the main factor for achieving a normal PBM in healthy individuals is genetically determined [6], PBM may be a major determinant of bone mass later in life $[6,7]$, and it is critical to maximize the potential of reaching the most optimal value through management of potential factors contributing to bone loss and osteoporosis. Environmental factors (e.g., sex hormones, calcium and vitamin D dietary intake, medication use and sedentary lifestyle) account for approximately $25 \%$ of the variance in PBM [6]. Furthermore, while PBM is typically attained by 30 years of age, approximately $90 \%$ of PBM is reached by the age of 18 years in females and 20 years in males [8].

The greatest loss of bone and mineral occurs due to genetic defects, such as osteogenesis imperfecta (OI) and X-linked hypophosphatemic rickets (XLH), but enzymatic defects such as hypophosphatasia and homocystinuria, and disorders of copper transport, such as Wilson's disease and Menkes' kinky hair syndrome, are also conditions that can lead to severe bone demineralization. Other rare causes of $\mathrm{PO}$ include idiopathic juvenile osteoporosis (IJO) [1,2], the osteoporosis-pseudoglioma syndrome, and the juvenile and early-onset Paget's disease [9].

Secondary causes of $\mathrm{PO}$ are much more common in children [1,2]. Secondary osteoporosis is typically multifactorial and it occurs as a consequence of systemic inflammatory diseases, which affect bone metabolism and endocrinopathies that act via altered hormone levels [2]. Furthermore, other diseases, such as various eating disorders, GI tract diseases determining malabsorption, or hepatobiliary diseases, may cause a reduction of bone density and quality. Finally, any cause of prolonged immobilization, such as cerebral palsy (through a reduction of mechanical stress on bone), inhibits osteoblast-mediated bone formation and accelerates osteoclast-mediated bone resorption, leading to a decreased BMD. Neoplastic disorders (especially leukemia and lymphoma, but also many solid tumours) and medications (glucocorticoids [GCs], anticonvulsants, aromatase inhibitors, lithium and heparin) are also important. Finally, decreased dietary intake of calcium or

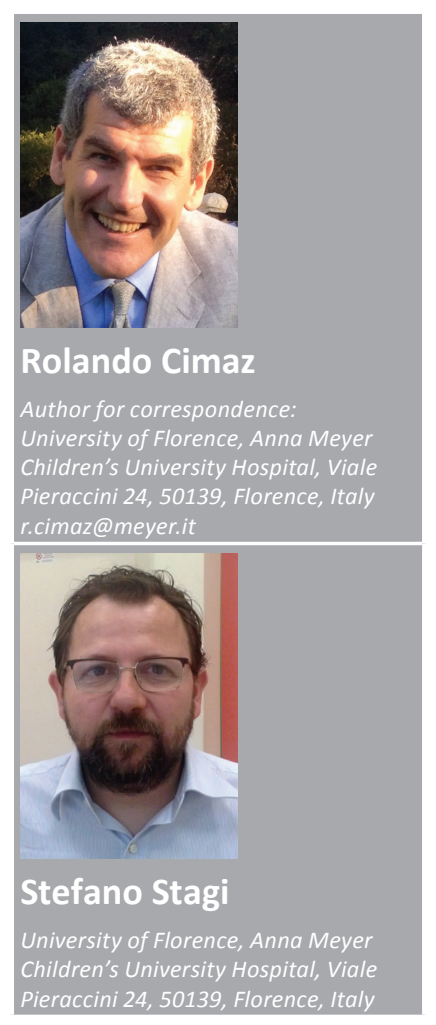

Future $\mathrm{fSg}$ 
vitamin $\mathrm{D}$ deficiency may be additional risk factors for PO [1,2].

In the case of inflammatory disorders, IL-1 $\beta$ and IL- 6 stimulate the osteoblast to produce RANKL, stimulating bone marrow stem cells to differentiate into osteoclasts, hence increasing bone resorption [9].

"Secondary osteoporosis is typically
multifactorial and it occurs as a consequence
of systemic inflammatory diseases, which
affect bone metabolism and endocrinopathies
that act via altered hormone levels."

With regard to pediatric rheumatic diseases, much data incontrovertibly showed that juvenileonset systemic lupus erythematosus patients are more prone to suffer from decreased BMD $[1,7,10]$, with a prevalence of osteopenia and $\mathrm{PO}$ of 37.5 and $20.3 \%$, respectively [10]. Multiple causes, involving both disease- and nondisease-related factors, have been proposed as main reasons for bone loss, including reduced physical activity, limited exposure to sunlight, systemic inflammation, GCs, delay in pubertal development, inadequate dietary intake of calcium and vitamins, use of cytotoxic drugs and disease severity, especially nephritis. Similar findings have been reported for dermatomyositis, even if, in this condition, the relatively reduced mobilization in myositis may also play a role $[1,2]$.

Children and young adults with juvenile idiopathic arthritis showed a decreased BMD [1,7], particularly in polyarticular and systemic groups [7], with a reduced PBM and an increased risk of developing adult osteoporosis [1,2,7]. Disease severity, inflammatory mediators and GCs have a definitive pathogenetic role for low BMD and reduced PBM [1,7].

\section{Management of pediatric osteoporosis}

Since the cause of bone loss is frequently multifactorial and the exact pathogenic mechanism of PO has not been clearly established in many conditions, it is difficult to proceed with rational treatments and prevention. For example, nutritional supplements in malabsorption or appropriate antibiotic therapy in cystic fibrosis may be useful in the management of bone loss in children and young adults with these conditions. At the same time, dietary interventions can improve bone health [11], and physical activity and exercise during childhood have been identified as primary methods of preventing PO and enhancing skeletal development [12].
Therefore, it is evident that a multidisciplinary approach will often be required for the correct management of PO. In addition to a pediatrician and a pediatric rheumatologist or endocrinologist, familiar with OP management, an important part of this team are radiologists, who should be experienced in undertaking bone density scans in children. Other healthcare professionals, such as physiotherapists and occupational therapists, orthopedic surgeons and specialist nurses also play a role.

To begin with, we must ask what therapies are currently available to treat or prevent bone loss in children. The number of drugs to treat bone fragility in children is limited and most have never been established as safe or effective in randomized controlled trials.

Furthermore, while in adults the guidelines for the treatment of osteoporosis are widely accepted, limited data for children and adolescents with PO makes it harder to set clear guidelines for the pediatric population. Moreover, there are few guidelines on how to use bone-active drugs in children and a consensus is needed among pediatric caregivers as to what conditions would most benefit from which drug [1].

With regard to genetic diseases, few specific treatments are available, short of gene therapy or stem cell transplantation. Exceptions to this appear to be the use of intravenous bisphosphonates in OI [2] or the use of phosphate and 1,25-dihydroxyvitamin D in XLH [2].

For conditions in which bone resorption is the primary defect, treatment with an antiresorptive agent would be the most appropriate option; however, for conditions in which lack of new bone formation is the predominant finding, use of anabolic agents should be considered along with appropriate management of the underlying condition.

\section{"...it is evident that a multidisciplinary approach will often be required for the correct management of pediatric osteoporosis."}

Vitamin D and calcium are important for maintaining adequate bone homeostasis and facilitating bone remodeling and growth. Even if in the presence of vitamin D deficiency and/or poor dietary calcium intake it is appropriate to replace such deficits, routine calcium and vitamin $\mathrm{D}$ supplementation is not recommended. In a meta-analysis, a reduced change in BMD was showed after calcium supplementation in healthy children [13]. 
In recent years, reduced sun exposure and a decrease in outdoor activities, in addition to an increased use of sunscreen and an increasingly lower time devoted to physical activity, are among the causes of decreased vitamin D levels in the general population.

In a large group of pediatric patients with osteoporosis or osteopenia, decreased levels of 25-hydroxyvitamin D were detected [14]. Although no direct connection with fracture risk may be found, vitamin D supplementation in children with osteopenia and PO is advisable and may indeed reduce morbidity. Low vitamin D levels, associated with decreased BMD levels and high parathyroid hormone levels, were found in juvenile idiopathic arthritis patients treated with GCs $[1,2]$. The recommended daily consumption of vitamin D may not be sufficient for patients undergoing bone-affecting treatments and for those suffering from primary bone disorders.

Recent data strongly suggests that physical activity in childhood is one of the most powerful preventive strategies against osteoporosis [12]. Weight-bearing physical activity during childhood and puberty affects bone mass and formation [12]. More and more data are available that support the importance of physical activity during childhood independently of exercise interventions for achieving optimal bone mass into young adulthood [12].

\section{"In young subjects ... one standard deviation increase in peak bone mass would reduce fracture risk by as much as 50\%."}

Many different physical activities, in particular those characterized by a considerable loading magnitude applied at a fast rate, have proven to exert the greatest osteogenic effect on the growing skeleton [12]. So, weight-bearing activities, such as jumping, appear to determine the greatest benefits to bone mineralization and structure during development. In young subjects, significant skeletal benefits are shown when the exercise stimulus is delivered for a sufficient time, highlighting the critical role to acquire an early active lifestyle to promote life-long skeletal health [12]. For example, Bonjour and colleagues have quantified that one standard deviation increase in PBM would reduce fracture risk by as much as 50\% [15].

Bisphosphonates are used as the principal treatment of bone fragility in genetic forms such as OI. Bisphosphonates have also been studied as a potential preventive measure in other diseases; for example, children with cerebral palsy have a $89 \%$ increase in BMD compared with $9 \%$ in controls $[1,2]$. In children receiving treatment with GCs, BMD was shown to decrease, but this was not observed in those patients receiving alendronate $[1,2]$. In children with connective tissue disease [1,2], safety and increased $\mathrm{z}$-scores were shown during treatment with alendronate. However, for primary prevention, the use of bisphosphonates in children receiving corticosteroids for chronic disease is not yet recommended [2].

\section{"The recommended daily consumption of vitamin D may not be sufficient for patients undergoing bone-affecting treatments and for those suffering from primary bone disorders."}

The most effective anabolic agent in adults, recombinant human parathyroid hormone, is not approved for use in children. In fact, several concerns exist regarding its safety, especially in younger patients, because of the development of bone tumors, particularly osteogenic sarcoma in young rats $[1,2,16]$. However, recombinant human parathyroid hormone is given to hypoparathyroid children [16], although long-term followup studies have not been carried out to assess the risk of osteogenic sarcoma in this population.

\section{Conclusion}

In summary, children may be affected by both primary and secondary bone loss. Primary bone loss involves genetic mutations leading to fundamental defects in either collagen synthesis or bone mineral conservation. Secondary bone loss results from the body's response to a variety of acute and chronic conditions and/or treatment. Since the goal is to improve pediatric bone health, which has lifelong implications, a correct evaluation of the etiology and pathogenesis of diseases, and the role of diseases and/ or treatments on bone health, are mandatory. A healthy lifestyle, physical activity and nutritional supplementation, such as calcium and vitamin D (when necessary), are first-line treatments. New medications, such as biphosphonates, are recommended in severe cases.

Financial \& competing interests disclosure

The authors have no relevant affiliations or financial involvement with any organization or entity with a financial interest in or financial conflict with the subject matter or materials discussed in the manuscript. This includes employment, consultancies, honoraria, stock ownership or options, expert testimony, grants or patents received or pending, or royalties.

No writing assistance was utilized in the production of this manuscript. 


\section{References}

1 Cimaz R. Osteoporosis in childhood rheumatic diseases: prevention and therapy. Best Pract. Res. Clin. Rheumatol. 16(3), 397-409 (2002).

2 Zhang C, Liu Z, Klein GL. Overview of pediatric bone problems and related osteoporosis. J. Musculoskelet. Neuronal Interact. 12(3), 174-182 (2012).

3 Gafni RI, Baron J. Overdiagnosis of osteoporosis in children due to misinterpretation of dual-energy x-ray absorptiometry (DEXA). J. Pediatr. 144(2), 253-257 (2004).

4 Zemel BS, Kalkwarf HJ, Gilsanz V et al. Revised reference curves for bone mineral content and areal bone mineral density according to age and sex for black and non-black children: results of the bone mineral density in childhood study. J. Clin. Endocrinol. Metab. 96(10), 3160-3169 (2011).

5 Bianchi ML, Baim S, Bishop NJ et al. Official positions of the International Society for Clinical Densitometry (ISCD) on DXA evaluation in children and adolescents. Pediatr. Nephrol. 25(1), 37-47 (2010).
6 Ralson SH, de Crombrugghe B. Genetic regulation of bone mass and susceptibility to osteoporosis. Genes Dev. 20(18), 2492-2506 (2006).

7 Stagi S, Masi L, Capannini S et al. Cross-sectional and longitudinal evaluation of bone mass in children and young adults with juvenile idiopathic arthritis: the role of bone mass determinants in a large cohort of patients. J. Rheumatol.37(9), 1935-1943 (2010).

8 Haapasalo H. Physical activity and growing bone. Development of peak bone mass with special reference to the effects of unilateral physical activity. Ann. Chir. Gynaecol. 87(3), 250-252 (1998).

9 Vega D, Maalouf NM, Sakhaee K. CLINICAL Review \#: the role of receptor activator of nuclear factor-kappaB (RANK)/RANK ligand/osteoprotegerin: clinical implications. J. Clin. Endocrinol. Metab. 92(12), 4514-4521 (2007).

10 Compeyrot-Lacassagne S, Tyrrell PN, Atenafu E et al. Prevalence and etiology of low bone mineral density in juvenile systemic lupus erythematosus. Arthritis Rheum. 56(6), 1966-1973 (2007).

11 Johnston CC Jr, Miller JZ, Slemenda CW et al. Calcium supplementation and increases in bone mineral density in children. N. Engl. J. Med. 327(2), 82-87 (1992).

12 Gunter KB, Almstedt HC, Janz KF. Physical activity in childhood may be the key to optimizing lifespan skeletal health. Exerc. Sport Sci. Rev. 40(1), 13-21 (2012).

13 Winzenberg T, Shaw K, Fryer J, Jones G. Effects of calcium supplementation on bone density in healthy children: meta-analysis of randomised controlled trials. BMJ 333(7572), 775 (2006).

14 Bowden SA, Robinson RF, Carr R, Mahan JD. Prevalence of vitamin D deficiency and insufficiency in children with osteopenia or osteoporosis referred to a pediatric metabolic bone clinic. Pediatrics 121 (6), e1585-e1590 (2008).

15 Bonjour JP, Chevalley T, Ferrari S, Rizzoli $\mathrm{R}$. The importance and relevance of peak bone mass in the prevalence of osteoporosis. Salud Publica Mex. 51(Suppl. 1), S5-S17 (2009).

16 Linglart A, Rothenbuhler A, Gueorgieva I et al. Long-term results of continuous subcutaneous recombinant PTH (1-34) infusion in children with refractory hypoparathyroidism. J. Clin. Endocrinol. Metab. 96(11), 3308-3312 (2011). 